\title{
Variable adaptability of coordination polymers of cadmium(II) to external mechanical stimuli
}

\author{
M. Đaković, M. Pisačić \\ ${ }^{1}$ University of Zagreb, Faculty of Science, Department of Chemistry, Horvatovac 102a, Zagreb, Croatia \\ mdjakovic@chem.pmf.hr
}

Recently, the traditional way of perceiving crystalline matter as static and brittle has started to change, and nowadays we are witnessing a growing number of examples were crystals display a plethora of flexible response to a variety of stimuli. They were found to move, jump, split, flex, twist, curl, explode, or to display a salient behaviour under UV radiation or heating, but lately they were also found to respond to the applied external mechanical force [1]. Organic molecular crystals present a majority of examples of crystal adaptability to external stimuli, whilst metal-organic adaptable crystals are still quite rare. In the first report on mechanical flexibility of coordination polymers we have shown that crystals of a family of Cd(II) coordination polymers are capable of displaying not only exceptional mechanical elasticity but also variable flexible responses to applied external pressure [2]. They can actually differently tolerate exerted force and the different tolerability is a result of slight differences in the importance of intermolecular interactions in crystal packing.

We the aim to understand the feature more deeply and to shed light on the underlying principles of the phenomenon, we have recently discovered unprecedented difference in plasticity of crystals of closely related class of Cd(II) coordination polymers [3]. In addition to variable plasticity, crystals also display remarkable pliability and ductility, not hitherto observed for metal-containing molecular crystals, which we present herein. To understand the phenomenon and rationalize observations, in addition to micro-focus SCXRD and AFM, we have also performed a series of custom-designed experiments and complemented those with an in-depth theoretical analysis. The results pointed at intermolecular interactions as the crucial structural feature in determining the type and extent of these highly unusual mechanical responses of crystalline metal-based polymeric materials.

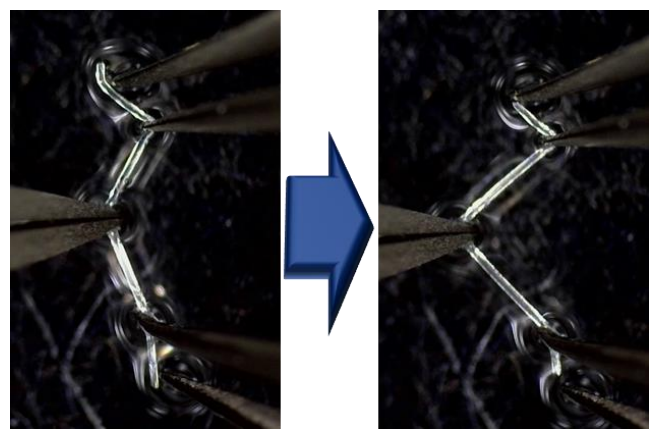

Figure 1. Pulling a crystal through sets of metal supporters; crystal immediately straighten as being upon pulled out.

[1] Commins, P., Israel Tilahun Desta, †Durga Prasad Karothu, $\uparrow$ Panda, M. K. \& Naumov, P. (2016) Chem. Commun. 52,13941.

[2] Đaković, M., Borovina, M., Pisačić, M., Aakeröy, C. B., Soldin, Ž., Kukovec., B.-M., Kodrin, I. (2018) Angew. Chem. Int. Ed. $130,15017$.

[3] Pisačić, M., Biljan, I., Kodrin, I., Popov, N., Soldin, Ž., Đaković, M. Chem. Mat. accepted.

Keywords: crystals flexibilty, extrnal mechanical stimuli, coordination polymers, intemolecular interactions, micro-focus SCXRD

This work has been fully supported by the Croatian Science Foundation under Project IP-2019-04-1242. 